\title{
Numerical prediction of the propagation of branched fatigue cracks
}

\author{
M.A. Meggiolaro ${ }^{\mathrm{a}, *}$, A.C.O. Miranda ${ }^{\mathrm{b}}$, J.T.P. Castro ${ }^{\mathrm{a}}$, L.F. Martha ${ }^{\mathrm{b}}$ \\ ${ }^{a}$ Mechanical Engineering Department, Pontifical Catholic University, Rio de Janeiro, RJ 22453-900, Brazil \\ ${ }^{b}$ Civil Engineering Department, Pontifical Catholic University, Rio de Janeiro, RJ 22453-900, Brazil
}

\begin{abstract}
A specialized finite element (FE) program is used to predict the propagation behavior of asymmetrically bifurcated cracks, which can cause crack growth retardation and arrest. The branched crack propagation path and associated stress intensity factors (SIF) are obtained for several bifurcation angles. It is found that very small differences between the branch lengths are enough to cause the shorter one to eventually arrest due to shielding effects. The SIF of the longer crack branch is also reduced due to the deflections, but it returns to the original non-bifurcated value as the crack propagates away from the influence of the (arrested) shorter branch. It is verified that crack bifurcation may provide an alternate mechanistic explanation for overload-induced crack retardation.
\end{abstract}

Keywords: Fatigue; Crack propagation; Crack path; Branched cracks; Overloads; Finite elements

\section{Introduction}

Fatigue cracks tend to propagate in mode I in both brittle and ductile solids, curving their paths if necessary to avoid rubbing their faces. Such cracks can significantly deviate from their mode I growth direction due to the influence of overloads, see Fig. 1. Since the stress intensity factors (SIF) associated to deflected or branched fatigue cracks can be considerably smaller than that of a straight crack with the same projected length, such deviations can cause retardation or even arrest of crack growth. However, it is generally recognized that the propagation behavior of a branched crack is a very difficult problem to develop accurate analytical solutions [1-4]. Therefore, numerical calculations provided by, for instance, finite element (FE) software are usually the only means to predict such retardation effects. Analytical solutions have been obtained for kinked and symmetrically branched cracks ( $b=c$ in Fig. 1); however, there are very few results available for the real case of bifurcated cracks with different branch lengths $(b>c)$. The study of asymmetrically branched cracks is a very important issue, since very small differences between the $b$ and $c$ lengths are enough to cause the shorter branch to arrest as the larger one propagates reaching its (approximately) pre-overload SIF and growth rate. This typical propagation behavior has been observed in many structural components,

*Corresponding author. Tel.: +55 (21) 3114-1638; Fax: +55 (21) 3114-1165; E-mail: meggi@alum.mit.edu

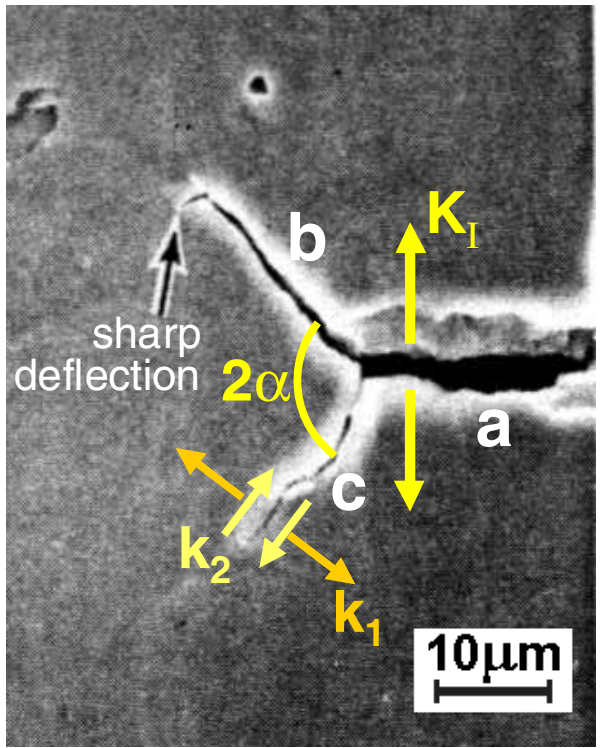

Fig. 1. Bifurcated crack geometry and associated nomenclature (adapted from [7]).

e.g. on a branched crack on an aircraft wheel rim made of 2014-T6 aluminum alloy [5].

To predict the path of a branched crack and to calculate the associated modes I and II stress intensity factors (SIF), an interactive graphical program named Quebra2D is 
used. This program simulates two-dimensional fracture processes based on a finite-element (FE) self-adaptive meshgeneration strategy, using appropriate crack tip elements and crack increment criteria. It has been validated through experiments on several modified CT specimens and from comparisons with analytical solutions for kinked cracks [6]. In the next section, this FE software is used to calculate the propagation behavior of bifurcated (branched) cracks.

\section{Propagation of branched cracks}

The propagation of branched cracks is studied using a FE model of a standard CT specimen with width $w=32.0$ mm and crack length $a=14.9 \mathrm{~mm}$, with a bifurcation at the crack tip with branch lengths $b=11 \mu \mathrm{m}$ and $c=10$ $\mu \mathrm{m}$ (see Fig. 1). The bifurcation angles $2 \alpha$ considered in this study vary between $60^{\circ}$ and $150^{\circ}$, which are characteristic values for experimentally obtained overload-induced branched cracks [7].

To calculate the propagation path in the Quebra2D program, three criteria are used for the numerical computation of the crack incremental growth direction in the linearelastic regime: the maximum circumferential stress $\left(\sigma_{\theta \max }\right)$, the maximum potential energy release rate $\left(\mathrm{G}_{\theta \max }\right)$, and the minimum strain energy density $\left(S_{\theta \min }\right)$ [6]. Bittencourt et al. [8] have shown that if the crack orientation is allowed to change in automatic fracture simulation, the three criteria basically provide the same numerical results. A fixed crack growth step of $3 \mu \mathrm{m}$ is considered for the propagation of the longer branch, calculated in the direction defined by the $\sigma_{\theta \max }$ criterion (which is the criterion adopted in this simulation due to its simplicity and to the availability of closed form solutions). However, due to the differences in the crack growth rate, a smaller growth step is expected for the shorter branch. This smaller step is obtained assuming a crack propagation law that models the first two growth regimes,

$\frac{\mathrm{d} a}{\mathrm{~d} N}=A \cdot\left(\Delta K-\Delta K_{t h}\right)^{m}$

where $A$ and $m$ are material constants and $\Delta K_{t h}$ is the propagation threshold. If $\Delta K$ and $\Delta K^{\prime}$ are respectively the stress intensity ranges of the longer and shorter branches, then the growth step $\Delta a$ of the shorter branch should be

$\Delta a=3 \mu \mathrm{m} \cdot\left(\frac{\Delta K^{\prime}-\Delta K_{t h}}{\Delta K-\Delta K_{t h}}\right)^{m}$

Interestingly, the ratio between the propagation rates of the two branches is independent of the material constant $A$. In this analysis, $\Delta K_{t h}$ and the exponent $m$ are assumed to be respectively $10 \mathrm{MPa} \sqrt{\mathrm{m}}$ and 3.0 , which are representative values for steels at low $R$ ratios.

It must be noted however that linear-elastic FE calculations can only lead to accurate solutions if the lengths of the crack branches ( $b$ and $c$ in Fig. 1) are significantly larger than the size scale of both the microstructure and the near-tip plastic (or process) zone. But as the crack branches grow further, the FE method can give a reasonable estimate of their behavior, in special for brittle or semi-brittle materials with small process zones. In addition, the growth of branched cracks is typically transgranular, as verified from optical microscope observations performed by Shi et al. [9], which is one of the requirements to allow for the simulation of fatigue behavior in an isotropic linear-elastic regime.

\section{Results}

Fig. 2 shows the mode I SIF $k_{1}$ at the tip of each crack branch (normalized by the mode I SIF $K_{I}$ of a straight crack) as a function of the length $b$ of the longer branch, measured along the propagation path. Because of the different crack lengths, the SIF at the larger crack tip is much higher than that at the shorter branch. Assuming $k_{1}$ to be the crack driving force, it can be seen from Fig. 2 that the longer branch reaches its minimum propagation rate right after the bifurcation occurs, returning to its preoverload rate as the crack tip advances away from the influence of the (probably arrested) shorter branch. Also, the mode I SIF of the shorter branch is progressively reduced due to shielding effects, resulting in crack arrest as the propagation threshold $\Delta K_{t h}$ is reached. Note that even small differences between the branch lengths (such as $1 \mu \mathrm{m}$ ) are sufficient to cause subsequent arrest of the shorter branch (Fig. 3). In addition, the retardation effect lasts longer for larger bifurcation angles (Fig. 3), not only because the associated mode I SIF is smaller, but also because the shielding effect is weaker since both branch tips are further apart, delaying the arrest of the shorter one.

Another interesting conclusion is that the initial propagation direction of the longer branch is always between $25^{\circ}$ and $30^{\circ}$ (with respect to the pre-overload growth direction), independently of the considered bifurcation angle $2 \alpha$. Therefore, for larger values of $2 \alpha$, a sharp deflection can be clearly noted in the beginning of the propagation. This deflection has been confirmed experimentally by Lankford and Davidson [7], who carried out overload fatigue crack tests on a 6061-T6 aluminum alloy in a scanning electron microscope using a special in-situ servo-controlled hydraulic loading stage, obtaining growth retardation caused by crack bifurcation. They have found that the bifurcated crack would grow only a short distance in the same direction of the overload-induced bifurcation before a sharp deflection in the crack path would occur, see Fig. 1. This deflection causes a sudden increase in $k_{1}$ almost immediately after the propagation begins (Fig. 2), resulting in a significantly smaller retardation effect if compared to simplistic predictions based on symmetrically branched crack solutions found in the literature. However, if the SIF of 

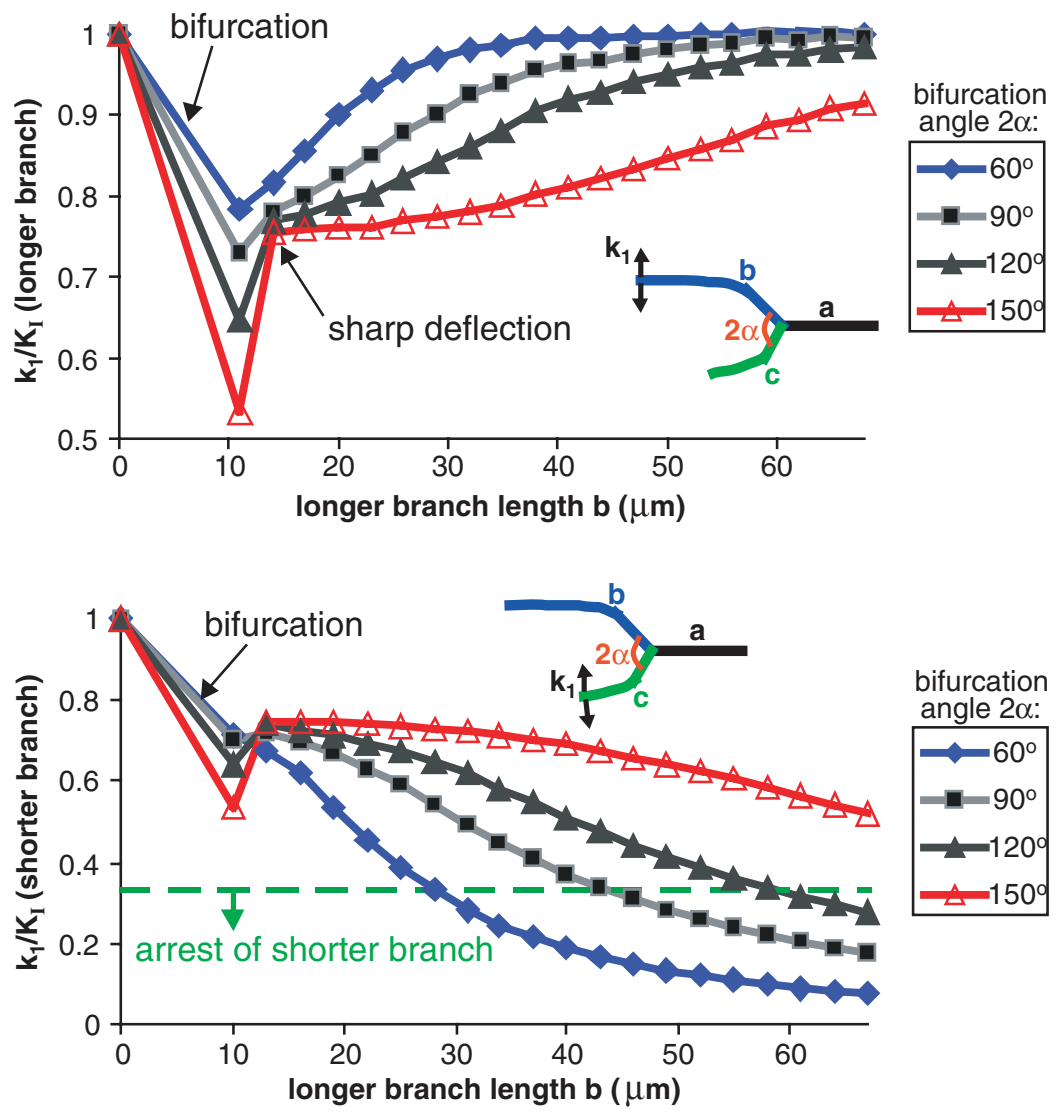

Fig. 2. Normalized mode I stress intensity factors for the longer (top) and shorter (bottom) branches of a bifurcated crack during its propagation.

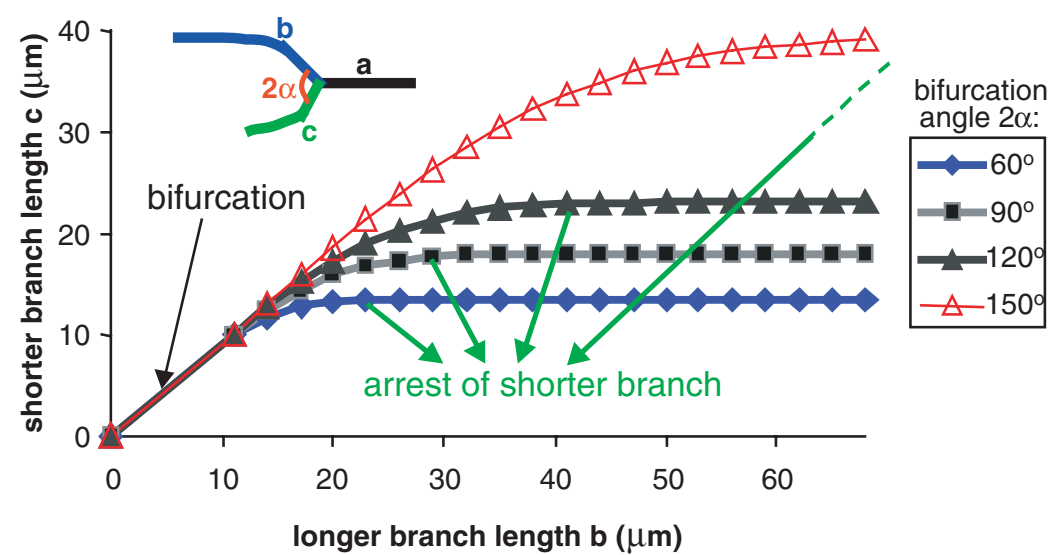

Fig. 3. Crack lengths of both branches of a bifurcated crack during its propagation, always resulting in crack arrest for the shorter branch.

both branches is below $\Delta K_{t h}$, then the entire crack arrests and therefore no sharp deflection has the chance to be developed.

Finally, Fig. 4 shows the propagation results for a bifurcated crack with angle $2 \alpha=150^{\circ}$. In this figure, the deformations are amplified to better visualize the crack path. Note that the crack path deviates from the original branch angles, deflecting from $\pm 75^{\circ}$ to approximately $\pm 28^{\circ}$. In addition, the originally shorter branch arrests after propagating to (only) $39 \mu \mathrm{m}$, while the longer branch 

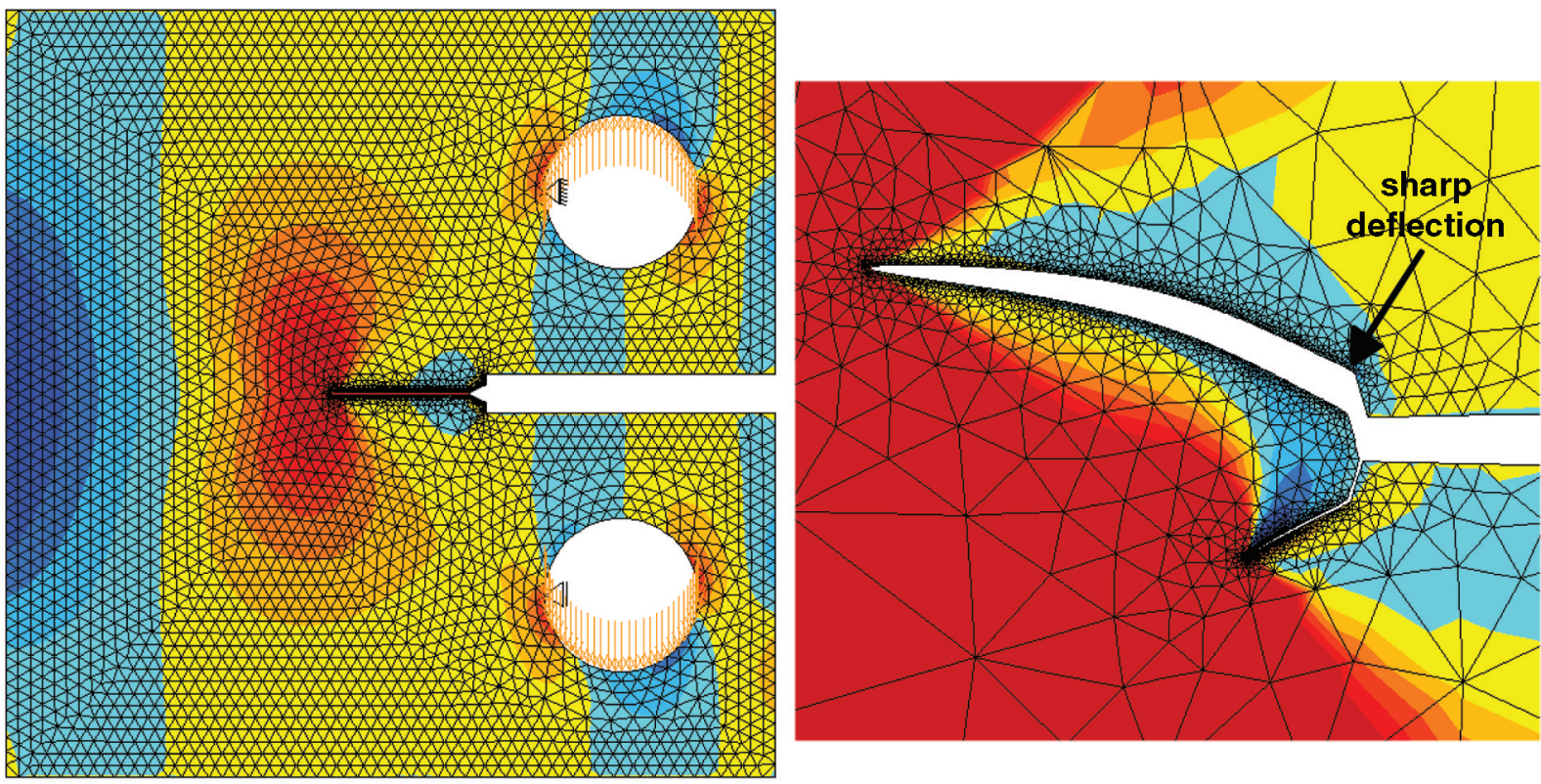

Fig. 4. Propagation simulation of a bifurcated crack on a CT specimen (left), with a close-up of the two original $11 \mu \mathrm{m}$ and $10 \mu \mathrm{m}$ branches with angle $2 \alpha=150^{\circ}$ (right).

returns to the pre-overload growth direction and SIF (even though the subsequent crack growth plane may be offset from the pre-overload one, see Fig. 4).

\section{Conclusions}

In this work, a specialized $\mathrm{FE}$ program was used to calculate the propagation path of bifurcated cracks, which can cause crack retardation or even arrest. It was shown that asymmetrically bifurcated cracks have a very different behavior than symmetrical ones, causing arrest of the shorter branch as the longer one returns to the pre-overload propagation conditions. From these results, it is found that crack bifurcation may provide an alternate mechanistic explanation for overload-induced crack retardation, in special to explain retardation under high $R$ ratios, since no crack closure would be detected in these cases. In addition, crack arrest can also be explained by this approach, happening when the mode I SIF $k_{1}$ of both branches are reduced to levels below the propagation threshold.

\section{References}

[1] Suresh S. Crack deflection: implications for the growth of long and short fatigue cracks. Metall Trans 1983;14A:23752385 .
[2] Suresh S. Micromechanisms of fatigue crack growth retardation following overloads. Eng Fract Mech 1983;18(3):577593.

[3] Suresh S. Fatigue of Materials. Cambridge University Press, 1998.

[4] Seelig Th, Gross D. On the interaction and branching of fast running cracks - a numerical investigation. J Mech Phys Solids 1999;47:935-952.

[5] Kosec B, Kovacic G, Kosec L. Fatigue cracking of an aircraft wheel. Eng Failure Anal 2002;9:603-609.

[6] Miranda ACO, Meggiolaro MA, Castro JTP, Martha LF, Bittencourt TN. Fatigue crack propagation under complex loading in arbitrary 2D geometries. In: Braun AA, McKeighan PC, Lohr RD (Eds), Applications of Automation Technology in Fatigue and Fracture Testing and Analysis. ASTM STP 2002;1411(4):120-146.

[7] Lankford J, Davidson DL. The effect of overloads upon fatigue crack tip opening displacement and crack tip opening/closing loads in aluminum alloys. Adv Fract Res 1981;2:899-906.

[8] Bittencourt TN, Wawrzynek PA, Ingraffea AR, Sousa JLA. Quasi-automatic simulation of crack propagation for 2D LEFM Problems. Eng Fract Mech 1996;55:321-334.

[9] Shi HJ, Niu LS, Mesmacque G, Wang ZG. Branched crack growth behavior of mixed-mode fatigue for an austenitic 304L steel. Int J Fatigue 2000;22:457-465. 